\title{
On Some Aspects of Linguistic Economy in English Academic Discourse
}

\author{
Astghik Chubaryan, Ruzan Karapetyan \\ Yerevan State University
}

$\mathrm{R}$ ecently scientists in CERN experiment have found some signs that certain particles travel faster than light. Of course this still needs thorough analysis and weighty confirmations, however it indicates the general trend of modern life very precisely - time today passes at a quicker rate than it used to. On second thoughts we all can memorize events which prove the accelerating speed of the modern flow of life. This phenomenon is reflected in practically every domain of life and surely language could not but be affected by this trend. The present article is devoted to the analysis of different genres of the scientific register of speech the qualities of which are predetermined and shaped by corresponding extralinguistic factors.

The nature, consequently the function, of science is such that it targets at obtaining and systematizing the knowledge of the world around us. Furthermore, it requires that the language, meant for the transfer of scientific knowledge, be compendious, laconic, logically constructed and be capable of transferring more information in less linguistic volume. Thus, the issue of linguistic economy, realized via the compression, is taken as central for this work.

The analysis of the empirical material really demonstrates the prior role of compression in scientific speech. It is worth mentioning that this priority is increasing, since we viewed this phenomenon on the diachronic plane as well. The following sources served as material for the empirical investigation for our analysis:

1) 42 pages of highly specialized papers from High Energy Physics, meant for professionals only;

2) A textbook for university students "Thermodynamics with Chemical Applications"; and

3) Posters presented at the international conference "Optical Liquid Crystals" held in Yerevan, September 25-30 2011

It should be mentioned that a special emphasis is placed on the regularities and differences of linguistic compression usage across genres, which proceeds from the functional characteristics of the given genre. Another point to be considered before getting down to the presentation of the data: linguistic compression is a very broad notion and can be realized on different levels and via various methods. The volume of the given work does not permit incorporation of all the aspects of this notion, so we will dwell upon absolute constructions and adverbial participles which are very effective in substituting subordinate clauses in scientific speech, for they are capable of absorbing more information in a lesser volume due to their structural peculiarities. Being very capacious these constructions transfer the same amount of information, as the corresponding subordinate clauses, more expeditiously. Present below are the examples: 
1. This looks like a kind of brane at $t=0$, with Neumann boundary conditions for the field $\mathbf{C}$.

2. Writing the metric as a deformation of the flat space metric $g_{\mu v}=\eta_{\mu v}+h_{\mu v}$, imposing the gauge conditions $h_{00}=h_{i 0}=h_{i i}=0$ and linearizing the Bach tensor,one can write the general solution as $(-\mathrm{ij}+\mathrm{k}(\mathrm{i} \zeta \mathrm{j}))-\mathrm{ik} 0 \mathrm{t}^{\wedge}-\mathrm{ij} \_\mathrm{eik.x}, \mathrm{k} \mu \mathrm{k} \mu=0$.

3. With I an interval of the time-axis $\left\{x_{-} h t, x 1, \ldots x d i: x 1=\cdots=x d=0\right\}$ we setA0(I) $\mathrm{A}(\mathrm{OI})$ where $\mathrm{OI}$ is the double cone $\mathrm{I} 00_{-} \mathrm{Rd}+1$, the causal envelope of $\mathrm{I}$.

4. Since the equation for $\mathrm{C}$ is conformal invariant we simply can consider it in flat space. Thus, dropping the denominator in (2.3) we get the flat space metric.

5. Thus, we often use ideal-gas heat capacities, these being different for different gases and functions of temperature only.

6. The third axiom does the entropy function what the first axiom does for the internal energy, asserting its existence and providing a relationship which connects it with measurable quantities.

The examples demonstrate the effectiveness of these constructions for scientific speech, but at the same time they show that the ellipsis of certain grammatical categories requires additional efforts on the part of readers/listeners to interpret the information correctly. This is connected with the level of professional preparedness of the readers/listeners and must definitely be taken into consideration by speakers/writers. In the examples provided the interconnection of the participial clauses with the main clause is quite ambiguous for non-professionals (temporal, conditional, instrumental, of cause-effect and so on), whereas in science these nuances in meaning are determinative.

Now let us present how the linguistic compression is reflected in each of the genres.

1) In the scientific papers examined we found 81 cases of Absolute constructions and adverbial participles at 42 pages only. This is quite a high concentration, especially in terms of the adequate interpretation of the information. This can be explained by the specificity of the genre of scientific paper itself, which is meant for a narrow circle of professionals who have detailed and complete idea concerning the topic under discussion. Thus, a compressed transfer of information can in no way affect the correct perception of the content, consequently it is justified by extralinguistic factors. In other words, the principle of the economical use of linguistic means dominates over the necessity of high level of explicitness in this sphere and this, in its turn is reflected frequent use of the constructions discussed.

Another interesting fact is connected with diachrony. Analysis of the papers written in the years between 1985-91, revealed the following statistics: 29 cases of absolute constructions at 218 pages. Whereas, in the papers analyzed for this work we found 35 cases of absolute constructions use at only 42 pages. This is mere statistics, however it might be assumed that the increasing demand of the time to be more concise and laconic is traced in this statistics.

2) The second source examined is a textbook for university students. Here we found 21 cases of absolute constructions and adverbial participles at 65 pages. As compared to scientific papers a significant decline of linguistic means fulfilling the function of 
compression is observed in this genre. This is predetermined by the necessity to be as explicit as possible, since the utmost goal of textbooks is to teach. As is seen the extralinguistic requirements of the genre in questions are directly proportional with the statistics. Thus, it can be supposed that the principles of linguistic economy in the given case are not weighty enough to regulate the use and functioning of linguistic units proper.

3) And the third genre analyzed is posters. This area has very little been investigated and requires a comprehensive insight, so we looked into the problem of narration efficiency both in terms of structural organization of posters and linguistic compression. Posters presented at the international conference "Optical Liquid Crystals" held in Yerevan, September 25-30 2011 served as material for investigation. We studied approximately 15 posters by Italian, Canadian, Taiwanese, Russian and Armenian scholars. It is to be stipulated that herein we present only certain preliminary observations, since accurate results and rigid assumptions require an in-depth, all-encompassing analysis of different linguistic levels.

The first point to mention is that in posters the cases of linguistic compression via adverbial participles and absolute constructions are practically absent. All in all we encountered just 2 cases of sentences with adverbial participles. Perhaps it might be accounted by the fact that in posters simple and concise sentences are more favoured, where the information is contracted to the highest possible extent. From the material analyzed it could be concluded that the data in posters is presented more schematically, either in graphs and figures or in points. These were the first two striking modes for information compression in posters, at least this is what we observed at this level of analysis. This is true practically for all the poster conclusions we examined. Here is an example:

\section{Conclusions}

- Artifact-free, compact "portable" CD spectrograph based on a cycloidal optical axis grating

- The diffraction properties of the cycloidal grating allow for real-time CD

- Extreme simplicity of the method: only the intensities I1 or,

\section{Perspectives}

- ECP range 190-360nm ask for UV transparent LC and KM

- Extend the method to circularly Luminiscence (CPL) and vibrational optical activity (i.e. ROA)

An interesting fact to mention and of course this should be taken into consideration when teaching Academic writing to Armenian students. All the posters by Armenian scholars were extremely wordy, long and obviously these were extracts from scientific papers, but the genre of posters does not permit it due to the space restrictions. So the principle of linguistic economy must be taught to students for them to observe the internationally-accepted norms. 
And to sum up, it should be mentioned that there are still numerous issues to be studied in the genre of posters on all the linguistic strata, because in posters the principle of linguistic compression is much more pertinent than in the other two sources discusses above.For now, we can state that the two evident ways for compressing information in posters are schematic presentation of data and simple sentences presented in points.

\section{References:}

1. Halliday, M.A.K.; Matthiessen, Ch. (1997) Systemic Functional Grammar. A First Step into the Theory.

2. Halliday, M.A.K. \& Martin, J.R. (1993) Writing Science: literacy and discursive power. London: Falmer Press.

3. Leech, G.; Hundt, M. (2009) Change in Contemporary English. A Grammatical Study. New York: CUP.

4. Chubaryan, A.; Karapetyan, R. (2004) Absolutniye konstruktsii kak sredstvo lingvisticheskoi ekonomii $v$ nauchnoi proze. // Foreign Languages for Special Purposes. N 4. Yerevan: YSU Press.

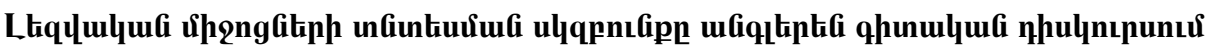

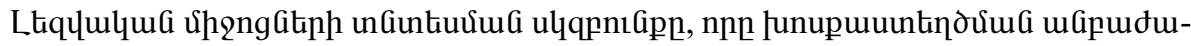

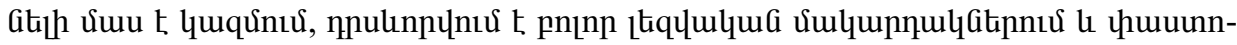

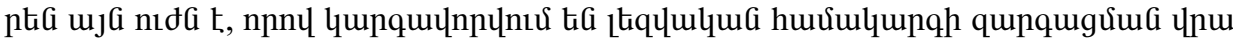

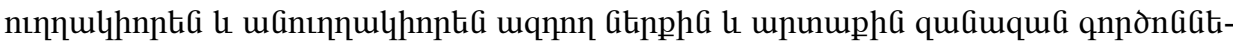

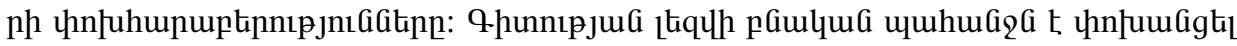

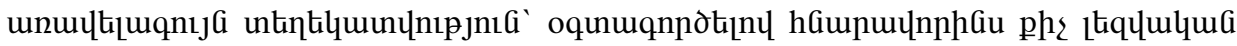

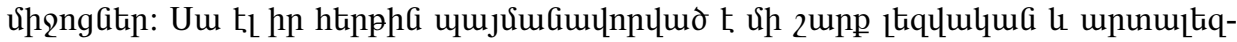

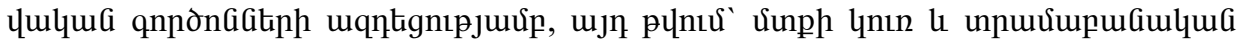

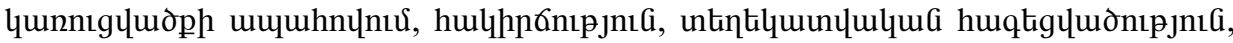

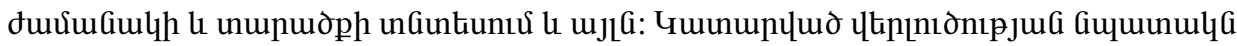

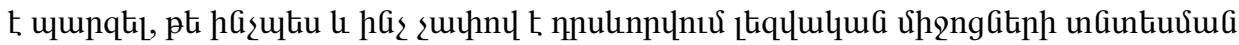

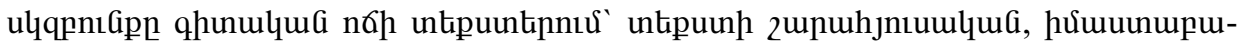

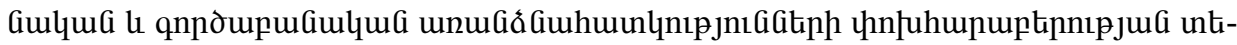
uuGlujnıGihg: 